\title{
Levosimendan for amyotrophic lateral sclerosis
}

Muscle weakness is the main characteristic of amyotrophic lateral sclerosis, which eventually leads to functional impairment and respiratory insufficiency. Progressive weakness derives from a loss of motor units and active muscle fibres. Striated muscle contraction depends on the interaction between intracellular calcium and the protein troponin. Troponin is made up of three subunits-troponin C is especially important for binding calcium and promoting a dynamic interplay between troponin and tropomyosin, with consequent muscle contraction. ${ }^{1}$ It has been suggested that a drug able to increase the affinity of troponin $C$ to calcium (a calcium sensitiser) would stimulate stronger contractions. $^{2}$ In patients with amyotrophic lateral sclerosis, a stronger contraction of surviving muscle fibres might compensate the weakness originating from the reduced number of motor units.

Levosimendan is a drug developed and approved in the EU to treat severe heart failure by intravenous administration. Its principal pharmacological

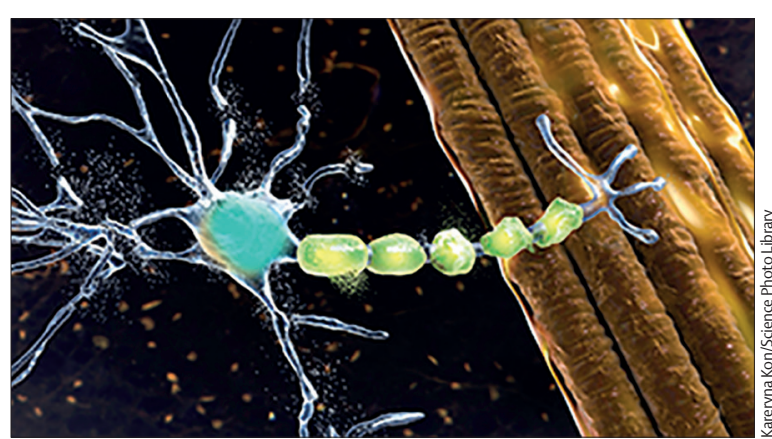


effect is to increase cardiac contractility by calcium sensitisation of troponin C. Because of the positive action of the drug on the neuromechanical efficiency and contractile function of the diaphragm in healthy controls, levosimendan was considered potentially useful for treating amyotrophic lateral sclerosis, a disease characterised by progressive upper and lower motor neuron deficits. ${ }^{3}$ This hypothesis was tested in the phase 2 LEVALS trial, ${ }^{4}$ in which the drug was given orally. The primary endpoint of that trial was not met, but in a post-hoc analysis, a positive effect on supine slow vital capacity (SVC) was found at 12 weeks. In another trial ${ }^{5}$ of a different drug (tiramsentiv) with a similar mechanism of action, the findings were also negative as tiramsentiv did not diminish the SVC rate of decay in the treatment group. ${ }^{5}$

In The Lancet Neurology, Merit Cudkowicz and colleagues present findings of the REFALS study, which was done at 99 centres across 14 countries, in Australia, Europe, and North America. ${ }^{6}$ In this randomised trial, a large cohort of patients with amyotrophic lateral sclerosis received either oral levosimendan $(n=329)$ or placebo $(n=167)$ over a 48-week period. The primary endpoint was change in supine SVC at 12 weeks from baseline (estimated treatment difference $0.26 \%, 95 \% \mathrm{Cl}$ -2.03 to $2.55, p=0.83$ ), which did not show any effect of levosimendan. The key secondary endpoint was the combined assessment of function and survival (CAFS), incorporating the revised ALS Functional Rating Scale (ALSFRS-R), which was assessed at 48 weeks, also showing no benefit of the study drug (least squares mean change from baseline 10.69, $95 \% \mathrm{Cl}-15.74$ to $37 \cdot 12$ ). Despite incorporating a testing hierarchy for the primary and secondary endpoints, several other secondary endpoints were also analysed by the authors at 48 weeks, including the ALSFRS-R respiratory subdomain score and time to ventilatory support. No prespecified trial endpoint showed a benefit of levosimendan versus placebo. Detailed analysis of ALSFRS-R in specific subgroups indicated some potential positive results of levosimendan, but the clinical relevance of these findings is dubious.

The rationale for the REFALS study is understandable since, although a drug such as levosimendan was not expected to cure amyotrophic lateral sclerosis or to halt disease progression, a positive effect on the decline of respiratory function would be important. Considering the trial duration, number of patients recruited, and the large number of centres involved, this trial must have been expensive and hard to manage. The authors were, therefore, assiduous in extracting a large amount of information, particularly regarding the extensive analysis of patient subgroups.

The decision to assess the primary endpoint at 12 weeks in the 48-week REFALS study was probably influenced by the results of the phase 2 LEVALS trial. ${ }^{4}$ In that study, levosimendan was reported to reduce the decline in supine SVC within a short period. The total duration of LEVALS was 6 months, and the trial followed a complex design, consisting of three 14-day crossover treatment periods separated by washout periods of 19-23 days. ${ }^{4}$ In their discussion, Cudkowicz and colleagues argue that analysing the primary endpoint at 12 weeks instead of 48 weeks in REFALS could obviate the effect of dropouts and patients on ventilatory support later in the trial. However, some complementary measurements would have been informative, the first of which would be to combine changes at 12 weeks with later evaluations of supine SVC. A second approach would be to add additional respiratory tests to ascertain changes in respiratory function not detected by supine SVC, such as nocturnal oximetry or capnography, and maximal inspiratory pressures. Taking into account the potential effect of levosimendan on diaphragm neuromechanical properties, adding neurophysiological studies would also have been opportune. Finally, including a wet biomarker, ${ }^{8}$ in particular blood neurofilaments, would have added information on potential correlations with respiratory function.

When designing the REFALS study, in their sample size calculation, Cudkowicz and colleagues proposed a small difference between the study groups as clinically significant (3.6\%). Considering this small effect, and that the trial did not meet any of the prespecified endpoints, levosimendan is probably ineffective in amyotrophic lateral sclerosis. Muscle cell physiology is different in skeletal muscles and the myocardium. Although levosimendan had some positive effects in the diaphragm of healthy controls, its effect on partly denervated skeletal muscle is unknown. Therefore, the results of the REFALS study should 
not necessarily prevent testing of other drugs with a similar mechanism of action, such as reldesemtiv (NCT04944784). Cudkowicz and colleagues should be congratulated on completing this difficult trial and for sharing their results. Amyotrophic lateral sclerosis researchers should recognise the effort put into and lessons taken from this trial, particularly regarding the study design. To find an effective drug to treat amyotrophic lateral sclerosis is a major challenge, and at the core of this challenge are the choice of a relevant compound and the use of the best methods for measuring treatment outcomes.

We declare no competing interests.

* Mamede de Carvalho, Michael Swash mamedemg@mail.telepac.pt

Instituto de Fisiologia, Instituto de Medicina Molecular, Faculdade de Medicina Univeridade de Lisboa, Lisbon 1648-028, Portugal (MdC, MS); Department of Neurosciences and Mental Health, Hospital de Santa Maria, Centro Hospitalar Universitário de Lisboa Norte, Lisbon, Portugal (MdC); Departments of Neurology and Neuroscience, Barts and The London School of Medicine, Queen Mary University of London, London, UK (MS)
1 Fusi L, Brunello E, Sevrieva IR, Sun YB, Irving M. Structural dynamics of troponin during activation of skeletal muscle. Proc Natl Acad Sci USA 2014; 111: 4626-31.

2 Lee JA, Allen DG. Calcium sensitisers. BMJ 1990; 300: 551-52.

3 Doorduin J, Sinderby CA, Beck J, et al. The calcium sensitizer levosimendan improves human diaphragm function. Am J Respir Crit Care Med 2012; 185: 90-95.

4 Al-Chalabi A, Shaw P, Leigh PN, et al. Oral levosimendan in amyotrophic lateral sclerosis: a phase II multicentre, randomised, double-blind, placebo-controlled trial. J Neurol Neurosurg Psychiatry 2019; 90: 1165-70.

5 Shefner JM, Cudkowicz ME, Hardiman O, et al. A phase III trial of tirasemtiv as a potential treatment for amyotrophic lateral sclerosis. Amyotroph Lateral Scler Frontotemporal Degener 2019; 0: 1-11.

6 Cudkowicz M, Genge A, Maragakis N, et al. Safety and efficacy of oral levosimendan in people with amyotrophic lateral sclerosis (the REFALS study): a randomised, double-blind, placebo-controlled phase 3 trial. Lancet Neurol 20: 821-31.

7 de Carvalho M, Swash M, Pinto S. Diaphragmatic neurophysiology and respiratory markers in ALS. Front Neurol 2019; 10: 143.

8 van den Berg LH, Sorenson E, Gronseth G, et al. Revised Airlie House consensus guidelines for design and implementation of ALS clinical trials. Neurology 2019; 92: e1610-23. 